\title{
A técnica de ERP no processamento de sentenças de crianças: uma revisão
}

\section{The ERP technique in children's sentence processing: a review}

Marília Uchôa Cavalcanti Lott de Moraes Costa

Universidade Federal do Rio de Janeiro, Rio de Janeiro, Rio de Janeiro / Brasil marilia@letras.ufrj.br

Resumo: A medição da ativação cerebral por meio da técnica de potenciais relacionados a eventos (ERP) tem sido valiosa para lançar luz sobre diversas cognições humanas. A linguagem é uma das cognições que têm sido estudadas com essa técnica de grande resolução temporal entre o estímulo apresentado e a ativação observada decorrente desse estímulo. Aárea de aquisição da linguagem tem se beneficiado especialmente dessa técnica, dado que é possível investigar relações entre dados linguísticos e a ativação cerebral sem a necessidade de uma resposta explícita, como apertar um botão ou apontar para uma imagem. O objetivo deste artigo é apresentar o estado da arte sobre o processamento de frases em crianças utilizando a técnica de ERP.

Palavras-chave: ERP/EEG; processamento de frases; aquisição da linguagem; N400; P600.

Abstract: The measurement of brain activation through the technique of event related potentials (ERP) has been valuable in shedding light on various human cognitions. Language is one of those cognitions that has been studied with this technique, which allows for more accurate temporal resolution between the stimulus presented and the time in which we observe an activation resulting from this stimulus. The area of language acquisition has especially benefited from this technique since it 
is possible to investigate relationships between linguistic data and brain activation without the need for an explicit response. The purpose of this article is to present the state of the art in children's sentence processing captured by the ERP technique.

Keywords: EEG/ERP; sentence processing; language acquisition; N400; P600.

Recebido em: 10 de dezembro de 2016.

Aprovado em: 4 de abril de 2017.

O objetivo deste artigo é revisar a literatura que utiliza a técnica de extração de potenciais elétricos de um traçado de eletroencefalograma (EEG/ERP) no campo de processamento de sentenças em crianças. Essa técnica tem sido utilizada por diferentes laboratórios, para medir diferentes modos de ativação cerebral relacionados a tarefas cognitivas e já demonstrou resultados importantes. Neste artigo, pretendo demonstrar resultados que atestam que crianças de 18 meses tem sensibilidade quanto à categoria de palavras e com 24 meses distinguem frases mal formadas daquelas bem formadas na língua que estão adquirindo. Estudos com crianças de até 4 anos são apresentados demonstrando a vasta competência linguística de crianças nessa faixa etária. Esse artigo também apresenta a técnica de ERP e os principais componentes encontrados em adultos, dessa forma é possível perceber as diferenças e semelhanças entre o processamento adulto e o infantil. Essa revisão procura contribuir com a área de aquisição da linguagem e neurociência da linguagem trazendo o estado da arte nessas duas áreas para o público brasileiro.

Para isso é relevante situar brevemente o leitor a respeito da técnica EEG-ERP. A eletroencefalografia (EEG) é uma técnica de imagem que mensura a atividade elétrica gerada por estruturas cerebrais (TEPLAN, 2002) na superfície do escalpo. Voltagens do escalpo refletem diretamente atividade neuronal. O EEG refere-se à atividade elétrica gravada continuamente da superfície do escalpo após ser capturada por canais, eletrodos de metal. O EEG é a mais antiga das técnicas de imagem. Hans Berger reportou a primeira gravação em 1929 (cf. LUCK, 2005, para informações históricas sobre a técnica). 
O EEG tem uma resolução temporal muito boa, na ordem de alguns milissegundos; ele é um procedimento não invasivo que pode ser utilizado tanto em adultos como em crianças. É relativamente barato quando comparado a outros sistemas, como a ressonância magnética funcional (fMRI) e o PET (Tomografia por emissão de Pósitrons), métodos hemodinâmicos, ou o magneto-encefalograma (MEG), e ele não requer pessoal especializado da área médica. Outro aspecto importante quando comparamos o EEG com métodos hemodinâmicos é que, diferente desses, o ambiente de testagem do EEG é silencioso, o que é ideal para estudos de linguagem que utilizam estímulos auditivos.

O EEG tem a desvantagem de ter uma menor resolução espacial, dado que, a eletricidade captada é gerada dentro do crânio e perpassa diversas barreiras como diferentes tipos de tecido cerebral e barreiras celulares até alcançar o escalpo. O ricochetear que a atividade elétrica sofre até chegar à superfície faz com que a ativação captada no escalpo e o local onde ela foi gerada não sejam análogos, ou seja, a fonte da atividade elétrica não está normalmente localizada logo abaixo do eletrodo onde está sendo feita a mensuração.

No sinal contínuo gravado pelo EEG, estão as respostas neuronais associadas a eventos sensoriais, linguísticos, e motores assim como atividade neuronal não relacionada e ruídos (TEPLAN, 2002). Para analisar as respostas elétricas correspondentes a um evento específico, a técnica de potenciais relacionados a eventos é utilizada (JOHNSON, 2001; LUCK, 2005). Essa técnica consiste em tirar uma média da atividade cerebral de muitos trials (item de um experimento) que correspondem à mesma condição experimental. A média permite que os pesquisadores eliminem o ruído e as atividades neuronais não relacionadas ao experimento. ${ }^{1}$ Ao calcular a média do sinal, assume-se que o sinal de interesse ocorra de maneira consistente através dos trials enquanto que o ruído é aleatório (LUCK, 2005).

No entanto, para garantir a exclusão do ruído indesejado e não do sinal relevante é necessário ter um grande número de itens experimentais para realizar as médias, termo conhecido como razão sinal-ruído ou relação sinal-ruído. Quanto maior o sinal, menor é a influência do ruído. Isso quer dizer que para obter uma onda ERP confiável em um experimento de linguagem padrão, a média deve ser feita com pelo

\footnotetext{
${ }^{1}$ Para facilitar a leitura chamarei toda ativação não relacionada ao experimento de ruído.
} 
menos 20-30 tokens diferentes dentro da condição experimental. Assim, quando falamos do ERP suscitado por uma palavra específica em uma condição particular, queremos dizer a atividade eletrofisiológica média em diferentes tokens do mesmo tipo (BROWN; HAGOORT; KUTAS, 2000).

O método de ERP pode identificar diretamente o potencial relacionado ao processamento de um estímulo. Ele é muito utilizado em estudos de aquisição da linguagem (OBERECKER et al., 2005; OBERECKER; FRIEDERICI, 2006; SILVA-PEREYRA et al., 2005a, 2005b; SILVA-PEREYRA et al., 2007; BERNAL et al., 2010; BRUSINI et al., 2016a, 2016b; COSTA, 2015) e processamento linguístico em geral (KUTAS et al., 1980; FRANÇA et al., 2004; GOMES; FRANÇA, 2013; GOMES, 2014; GOMES; FRANÇA, 2015). Outra vantagem de ERPs é que não há a necessidade de os participantes fazerem uma tarefa - o que torna essa tecnologia mais atrativa em populações mais jovens que podem participar de estudos de linguagem sem receber instruções (CONBOY et al., 2008). Na maioria dos estudos com ERP os voluntários recebem instruções para realizar uma tarefa apenas para mantê-los atentos, mas a atividade cerebral pode ser capturada sem nenhuma tarefa específica.

Após décadas de estudo, alguns componentes elétricos foram frequentemente observados - N170, ELAN, N400, P300, P600 - quando participantes processam estímulos específicos (como fala, faces, etc) e hoje eles são considerados marcadores de certos tipos de processamento realizado pelo cérebro. Há restrições para qualificar uma certa atividade cerebral encontrada por um paradigma experimental como um componente elétrico já muito conhecido. Existem critérios bem definidos para que uma dada ativação cerebral seja caracterizada como um N400 ou P600, por exemplo. A população de neurônios envolvida na atividade identificada precisa ser grande o suficiente e a atividade deles precisa ser sincronizada e coerente durante uma janela de tempo com duração suficiente; em nosso exemplo, a duração mínima seria $200 \mathrm{~ms}$. A definição de um potencial consiste de três dimensões da atividade gravada:

1. Latência (ms): o tempo em que um potencial começa, se desenvolve e desaparece;

2. Distribuição Espacial: a disposição dos eletrodos que exibem o efeito;

3. Voltagem: a polaridade do efeito (positiva ou negativa). 
Esses componentes são comumente nomeados levando em consideração a polaridade deles $(\mathrm{N}$ ou $\mathrm{P})$ e o número de milissegundos em que se observa tipicamente o efeito máximo (e.g.: N400, P600).

A literatura sobre aspectos linguísticos no cérebro já reúne um número considerável de trabalhos e há muitas hipóteses sobre como se dá o processamento linguístico. Poeppel e Embick (2005) e Marantz (2005) expressam as dificuldades e a importância da interseção entre a Linguística e a Neurociência (POEPPEL; EMBICK, 2005; MARANTZ, 2005). Desafios importantes que precisam de atenção são chamados por Poeppel e Embick de incompatibilidade de granularidade e incomensurabilidade ontológica. O primeiro conceito está relacionado com o fato de haver uma incongruência entre os elementos representacionais das duas disciplinas. Em geral a linguística trabalha com elementos bem detalhados, traços distintivos, morfema, sintagmas etc., enquanto que em abordagens neurocientíficas os termos são vistos de maneira mais ampla, dendritos, neurônios, coluna cortical. O segundo conceito está relacionado com o fato desses elementos das teorias linguísticas não poderem ser diretamente comparados com as unidades observadas pela Neurociência. Os autores apontam que esses problemas não são específicos da área da linguagem, mas são "problemas de interface" comuns aos estudos da cognição" (POEPPEL; EMBICK, 2005).

Apesar de a possibilidade de descobrir os mecanismos subjacentes ao processamento linguísticos ser empolgante, os elementos que eliciam ou afetam algum componente de ERP não se equiparam a uma função neurocognitiva. Por enquanto, a forma mais direta de observar um resultado de ERP é apresentar eventos altamente controlados que variam em apenas uma variável e compará-los entre muitos sujeitos. Se uma diferença for encontrada, então deve ser pelo fato de os estímulos terem alguma propriedade que o cérebro dos participantes "pôde diferenciar".

Cada estudo postula uma explicação que se encaixa aos dados e possivelmente a uma teoria, mas é apenas após um número de manifestações que seremos capazes de entender melhor o que os elementos descritos na literatura de ERP como componentes distintos quer dizer sobre os mecanismos subjacentes. A cada novo estudo entendemos mais sobre a linguagem, o cérebro e a técnica. Muitos anos após o primeiro estudo de ERP em linguagem, o debate ainda está aberto sobre o que significam para a linguagem os vários componentes de ERP encontrados e como integrar esses estudos com o corpo massivo de 
trabalhos já existentes em psicolinguística para alcançarmos os melhores modelos sobre processamento de linguagem. Como Pylkkänen e colegas afirmam:

In this endeavor, a cognitive model is crucial as without it, the cognitive neuroscientist does not know what to look for in the brain, what the nature of the relevant representations might be, or how the different components of a process might interact with each other." (PYLKKÄNEN; BRENNAN; BEMIS, 2011).

\section{Componentes comumente encontrados em experimentos de processamento de frases em adultos}

Muito já foi aprendido sobre o fato de anomalias linguísticas nos níveis semântico, morfológico e sintático eliciarem uma série de respostas de ERP com características diferentes. Estudos com anomalias sintáticas e morfológicas, por exemplo, têm demonstrado respostas qualitativamente diferentes de ERP aos diferentes tipos de violações. Abaixo faremos uma revisão destes tipos de componentes.

Como já foi mencionado, componentes de ERP são normalmente denominados de acordo com sua polaridade e latência. No entanto alguns componentes podem ser nomeados a partir de sua topografia. LAN (Left Anterior Negativity, negatividade anterior à esquerda) e ELAN (Early Left Anterior Negativity - negatividade anterior à esquerda precoce) são dois exemplos na área da linguagem. As duas ondas aparecem cedo e são eliciadas, de acordo com a literatura, por violações sintáticas. LAN e ELAN são, como o nome sugere, ondas negativas capturadas à esquerda nas áreas anteriores do cérebro. Essas duas ondas, analisadas como idênticas, diferem apenas em sua latência. O ELAN é muito precoce e aparece entre 150 e 200 milissegundos após o começo do elemento crítico; já o LAN se desenvolve entre 300-500 milissegundos. Estudos reportaram que o LAN e o ELAN opunham frases neutras, gramaticais, de frases contendo violações sintáticas: a morfossintaxe não estava adequada (concordância ou marcador de caso repetido) ou em sentenças contendo uma combinação de palavras inadequadas (e.g. uma palavra ou grupo de palavras aparecia em um contexto em que palavras de uma categoria sintática diferente eram esperadas - HAGOORT; LEVELT, 2009; HWANG; STEINHAUER, 2011). 


\section{O N400}

No final dos anos 70, Martha Kutas e Steven Hillyard estavam investigando a influência do contexto sentencial para o reconhecimento de palavras. Eles inicialmente pensaram que a manipulação do grau de previsibilidade do final de frases os levaria a observar o componente $\mathrm{P}^{2}$, que é normalmente relacionado à probabilidade de um certo estímulo aparecer. Os autores manipularam o final de frases, que variavam de congruentes até ininterruptas, mas, ao invés de uma onda posterior positiva, eles encontraram uma onda negativa. $\mathrm{Na}$ verdade, a única manipulação que deu o gatilho para um $\mathrm{P} 3$ foi o uso de finais congruentes, porém com fontes distintas, letras maiúsculas, na última palavra. Essa nova forma de onda foi chamada de N400 devido à sua polaridade e ao fato de seu pico ser observado em torno dos 400ms (KUTAS; HILLYARD, 1980). Em 1980, o primeiro artigo sobre o N400 foi publicado. Um par de exemplos desse trabalho foi "I like my coffee with cream and sugar." versus "I like my coffee with cream and socks." ". O segundo tipo de frase levaria a uma onda negativa $400 \mathrm{~ms}$ após o elemento crítico que nesse caso é a palavra final (sugar ou socks). Esse trabalho seminal foi feito com estímulos visuais, escritos. Naquele momento, os autores interpretaram a onda como uma resultante de uma violação semântica. Um grande número de trabalhos replicaram esse efeito estendendo os achados a outras modalidades (HOLCOMB; COFFEY; NEVILLE, 1992) e outros contextos linguísticos, incluindo pares de palavras e contextos discursivos mais amplos, assim como sentenças (OSTERHOUT; HOLCOMB, 1992; BERKUM; HAGOORT; BROWN, 1999; FRANÇA, 2002; FRANÇA et al., 2004; HÄNEL-FAULHABER et al., 2014).

Hoje, o N400 é descrito como uma deflexão negativa ampla do ERP que começa 200-300 ms após uma palavra ou sinal, no caso das línguas de sinais, ser apresentada por via auditiva ou visual e que tem seu pico depois de $400 \mathrm{~ms}$, aproximadamente, em eletrodos centro-parietais (cf. KUTAS; FEDERMEIER, 2011; LAU; PHILLIPS; POEPPEL, 2008). Pesquisadores observaram o N400 em um número variado de tarefas que variam de priming de palavras e processamento no nível

\footnotetext{
${ }^{2}$ Uma onda positiva que tem seu pico normalmente 300 milissegundos após o elemento crítico referida como P3.

${ }^{3}$ A primeira frase lê-se "Eu gosto do meu café com creme e açúcar" e a segunda "Eu gosto do meu café com creme e meias"
} 
da mensagem até o papel da atenção e consciência na compreensão da linguagem, diferenças de ativação entre hemisférios ativados pela memória semântica, e a construção de vocabulário no aprendizado de uma língua (OSTERHOUT; MCLAUGHLIN, 2006; KOTZ; HOLCOMB; OSTERHOUT, 2008), só para mencionar alguns trabalhos. O N400 também já foi usado para comparar diferenças entre populações - e.g., jovens vs. idosos, sadios vs. pacientes esquizofrênicos (KUPERBERG et al., 2006).

In general then, the N400 is thought to reflect the degree of ease or difficulty in retrieving stored knowledge associated with a potentially meaningful item from semantic memory, contingent upon both the characteristics of the stored item itself, as well as the contextual cues available. (KUTAS; DELONG, 2008, p. 157)

Apesar de uma resposta N400 ser majoritariamente associada a uma anomalia semântica, ela também já foi eliciada pela maioria de estímulos significativos - palavras faladas, escritas ou sinalizadas, nãopalavras pronunciáveis, e acrônimos conhecidos, mas também para sons não-humanos, faces, gestos, e cenas. Desde a primeira vez que a onda foi reportada, pesquisadores têm tentado explicar os mecanismos subjacentes relacionados ao N400. O componente já foi estudado a partir de diferentes ângulos e campos de investigação e mesmo assim ainda hoje há muitos aspectos que não são completamente compreendidos. De acordo com Kutas e Federmeier (2011), um ponto crucial da literatura de N400 é o panorama convincente de como a percepção, a atenção, a memória, e a linguagem em conjunto participam nos eventos neuronais responsáveis pelo N400 (KUTAS; FEDERMEIER, 2011). Apesar de não ser eliciado exclusivamente em estudos de processamento de linguagem, o N400 é uma ferramenta para investigar questões relacionadas ao curso temporal do processamento de linguagem. Um ponto permanece em discussão: exatamente qual(is) processo(s) o componente reflete?

\section{O P600}

O P600 foi descrito pela primeira vez em 1992 por Osterhout e Holcomb. É uma onda com voltagem positiva que apresenta sua amplitude máxima em torno de $600 \mathrm{~ms}$ após o estímulo crítico. Ele é eliciado tanto 
na modalidade visual quanto na auditiva. Ativa tipicamente eletrodos centro-parietais, mas alguns trabalhos já reportaram esse componente em eletrodos frontais (FRIEDERICI, 2002). O P600, também chamado de Syntactic Positive Shift - mudança sintática positiva (SPS), devido a sua natureza positiva e ao fato de ser eliciado por estímulos sintáticos (OSTERHOUT; HOLCOMB, 1992; FRIEDERICI; PFEIFER; HAHNE, 1993; HAGOORT; BROWN; GROOTHUSEN, 1993; OSTERHOUT; HOLCOMB; SWINNEY, 1994; GOMES; FRANÇA, 2013; GOMES, 2014; GOMES; FRANÇA, 2015).

Originalmente, o P600 foi interpretado como um reflexo de processos sintáticos. Ele foi observado em resposta a diferentes violações sintáticas, incluindo violações na estrutura sintagmática (OSTERHOUT; HOLCOMB, 1992; HAGOORT; BROWN; GROOTHUSEN, 1993), violações de subcategorização (OSTERHOUT; HOLCOMB, 1992; OSTERHOUT; HOLCOMB; SWINNEY, 1994), violações quanto ao número, tempo, gênero, e concordância de caso (COULSON; KING; KUTAS, 1998; NEVINS et al., 2007) e violações às restrições de dependência de longa distância (PHILLIPS; KAZANINA; ABADA, 2005). Devido à variedade de circunstâncias em que o P600 foi observado, ele foi interpretado como o reflexo de reparo sintático - e.g., (OSTERHOUT; HOLCOMB, 1992; FRIEDERICI, 1995), reanálise estrutural, ou integração sintática (GIBSON, 1998; KAAN et al., 2000; HERTEN; KOLK; CHWILLA, 2005). No entanto, nos últimos 10 anos a interpretação de que o P600 é especificamente relacionado a processamento sintático tem sido questionada (KUPERBERG; SITNIKOVA, 2003; KOLK et al., 2003; KIM; OSTERHOUT, 2005). Hoje já se discute o P600 como um componente que pode refletir tanto o componente sintático como semântico (BORNKESSEL-SCHLESEWSKY; SCHLESEWSKY, 2008; HOEKS; STOWE; DOEDENS, 2004; KUPERBERG, 2007).

Gouvea et al. (2010) estudaram todos os "estímulos P600" comumente descritos para um melhor entendimento da natureza dessa onda. Eles utilizaram um desenho experimental intra-sujeito com o máximo de estímulos similares para três tipos de configuração estruturais que havia sido discutido na literatura como "eliciadores de P600" - sentenças agramaticais, garden paths, e complexidade de dependências de longa-distância. O resultado foi que a topografia para garden paths sintáticos, violações de gramaticalidade, e preenchimento de dependências de longa-distância eram bastante semelhantes, mas 
as dependências com relação a palavras qu- tinham uma distribuição diferente, inicialmente mais anterior. A condição das dependências de qu- eliciaram um P600 de amplitude menor, e as condições com frases garden path e agramaticais apresentaram uma negatividade adicional e "o P600 posterior tinha uma latência retardada nas condições agramaticais (500-700 ms) do que nas condições com frases garden path (300-500 ms)" (GOUVEA et al., 2010).

Gouvea et al. (2010) sugerem que o P600 possa refletir um conjunto comum de processos que começam assim que informações suficientes foram acumuladas para iniciá-los. Sob essa visão, mesmo quando o P600 ocorre em latências distintas, ele ainda reflete os mesmos processos subjacentes, a diferença de latência refletiria mais ou menos o tempo necessário para completar os processos que acionam o P600.

Uma vez que a positividade que atinge picos de $600 \mathrm{~ms}$ é provocada por diferentes tipos de estímulos, ela levanta naturalmente a questão de se ela reflete um único mecanismo subjacente ou vários processos diferentes. Por enquanto, alguns pesquisadores discutiram possíveis maneiras de explicar o P600 como refletindo diferenças nos cálculos realizados por um único processo subjacente, mas ainda pode haver a possibilidade de que pequenas diferenças de topografia, tensão e tempo de pico sejam índices de diferentes mecanismos subjacentes.

\section{Estudos de linguagem ERP sobre processamento de sentenças em crianças pequenas}

Não há muitos estudos de ERP testando processamento de sentenças em crianças, por várias razões: (i) artefatos de movimento; (ii) dificuldade de colocação dos eletrodos/ touca de EEG; (iii) participação dos responsáveis; (iv) duração dos experimentos e dificuldade de encontrar estímulos atraentes. Como foi explicado anteriormente, uma das principais dificuldades para uma gravação de qualidade é o movimento. Solicita-se aos participantes adultos que não se movam e se abstenham de piscar em partes críticas do teste. Em contraste, você não pode pedir a uma criança pequena para ficar quieta, especialmente quando ela tem entre 14 a 36 meses - a idade exata quando você precisa estudar o início do processamento da sentença. E muito cansativo para eles permanecer no lugar por um longo tempo. A última barreira é a duração dos experimentos: embora o EEG seja a técnica ótima para estudos de 
crianças porque é silencioso e as crianças não têm que dar respostas abertas, o ERP exige a média de múltiplos trials em cada condição, o que torna as experiências geralmente muito longas para crianças (20-30 minutos). Se uma criança não participar do experimento por um período de tempo suficiente, o número de itens será muito baixo para obter médias estáveis. Para incentivar as crianças a permanecerem no experimento tempo suficiente, os estímulos precisam ser muito atraentes para elas. Todas essas barreiras podem explicar por que há tão poucos estudos com crianças pequenas na literatura ERP.

Uma última dificuldade com as crianças é que um cérebro em desenvolvimento não tem as mesmas propriedades de um cérebro totalmente desenvolvido 4 (PUJOL et al., 2006). Não é raro que os componentes de ERP de crianças sejam diferentes temporalmente, topograficamente ou quanto à polaridade. É difícil, portanto, usar o conhecimento que temos da literatura adulta para extrapolar o que deve ser encontrado em populações jovens (COCH, MITRA, GEORGE, 2012). As diferenças podem ocorrer em latência, duração e amplitude.

Embora haja muitos obstáculos na pesquisa de desenvolvimento de ERP, ainda existem alguns grupos ao redor do mundo que realizam essa empreitada. Encontramos estudos de ERP em diferentes domínios cognitivos e idades (KOVELMAN, 2012). No estudo da língua, a maioria dos estudos é feita em inglês, francês ou alemão. Existem muitos estudos em prosódia e fonologia (MILLS et al., 2004). Há evidências de que as crianças podem discriminar entre categorias de som, padrões de tonicidade (stress) (FRIEDERICI; FRIEDRICH; CHRISTOPHE, 2007) e unidades prosódicas, por exemplo. Os estudos em fonologia geralmente são realizados com bebês bem pequenos, que não se importam com as toucas; em muitos casos, os estudos podem ser feitos até mesmo quando o bebê está desatento à tarefa (por exemplo, distraído com bolhas de sabão enquanto ouve sílabas ou palavras) e às vezes até durante o sono (DEHAENE-LAMBERTZ; PENA, 2001).

Há também trabalhos sobre a segmentação da fala. $\mathrm{O}$ trabalho realizado com crianças de até 7 meses de idade apresentou evidência

\footnotetext{
${ }^{4}$ Cf. (SHAFER; GARRIDO-NAG, 2007) para uma revisão sobre as evidências neurobiológicas relativas ao desenvolvimento da linguagem e (POEPPEL; OMAKI, 2008) para uma discussão sobre as ressalvas e perspectivas do uso de abordagens ERP no desenvolvimento da linguagem.
} 
significativa de segmentação da fala. Os participantes mostraram uma polaridade positiva (KOOIJMAN et al., 2013). Os estudos foram conduzidos com técnica ERP para investigar o acesso lexical também. Os pesquisadores desenvolveram um paradigma apropriado para adultos e crianças, no qual o participante vê uma imagem de um objeto e ao mesmo tempo ouve um estímulo auditivo que é uma palavra correspondente ao nome do objeto ou não, ou é uma pseudopalavra que é fonotaticamente bem ou mal formada - em português poderíamos ter exemplos como baluga e btinica, respectivamente. Usando esse paradigma, Friedrich e Friederici (2004) observaram uma mudança de desenvolvimento entre as idades de 12 e 19 meses. Os efeitos de ERP em crianças de 19 meses foram relatados como bastante semelhantes aos dos adultos, isto é, um efeito N400 para as palavras incongruentes e pseudopalavras fonotaticamente possíveis, mas não para pseudopalavras fonotaticamente ilegais (FRIEDRICH, FRIEDERICI, 2004). No entanto, eles não encontraram uma negatividade aos $400 \mathrm{~ms}$ para 12 meses de idade. Diferentemente dos resultados encontrados com 12 meses de idade, em outro estudo realizado em 2010, os autores observaram um efeito N400 de priming com a mesma população. A ativação foi relacionada ao nível de produção de palavras de bebês. Os participantes foram divididos em dois grupos: um com alta produção de palavras precoces, e o outro com baixa produção de palavras precoces. Apenas o primeiro grupo teve o que Friedrich e Friederici interpretou como um N400 (FRIEDRICH; FRIEDERICI, 2010).

De acordo com Silva-Pereyra e colegas (2005b), a maior parte das crianças apresenta dois momentos de desenvolvimento sem igual após o primeiro ano de vida. O primeiro é uma aceleração na velocidade do crescimento do vocabulário a partir de 16 e 20 meses (SILVA-PEREYRA et al., 2005b). Aos 20 meses de idade, diferenças de ERP entre palavras conhecidas e não conhecidas se tornam lateralizadas e mais diferenciadas nas regiões temporal e parietal do hemisfério esquerdo, possivelmente refletindo o aumento de especialização dessas áreas para o processamento da linguagem (cf. os trabalhos do grupo de Debra Mills para uma revisão desses resultados). O segundo é no desenvolvimento da morfossintaxe das crianças, entre 24 e 30 meses de idade. Esse segundo momento é mais relevante para esse trabalho, dada a relação com o processamento de sentenças. 
Oberecker et al. (2005) e Oberecker e Friederici (2006) trabalharam no processamento de sentenças em crianças de 2 anos, de 28 meses e de 24 meses de idade, respectivamente. $\mathrm{O}$ estudo foi realizado em alemão. Eles testaram se nessa idade as crianças eram sensíveis às estruturas sintáticas de sua língua, como exemplificadas em (1), (2) e (3):

(1) Der Löwe brüllt

O leão ruge (ou está rugindo)

(2) Der Löwe im Zoo brüllt

O Leão no Zoo rugindo

(3) Der Löwe im brüllt

O leão no rugindo

As sentenças foram apresentadas a crianças em três condições - duas condições gramaticais e uma agramatical. As frases gramaticais tinham ou estrutura de DP (VP) como em (1) ou estrutura de DP [PP VP] como em (2). A condição agramatical tinha sentenças com descasamento de maiúsculas e minúsculas; o caso era marcado por uma preposição que tomava um nome ou adjetivo, mas as frases tinham um verbo em vez disso como em (3). Os resultados apresentados em Oberecker, Friedrich, Friederici (2005) demonstraram que os participantes de 2 anos e 8 meses exibiram o que os autores chamaram de ELAN e P600, enquanto que aqueles de 2 anos exibiram apenas um P600 (OBERECKER; FRIEDERICI, 2006). O padrão encontrado no grupo mais velho estava alinhado com o grupo-controle adulto que também exibiu um ELAN e P600. Os autores interpretaram esses achados sugerindo que os princípios de sintaxe são estabelecidos precocemente durante o desenvolvimento, uma vez que um P600 estava presente no grupo mais jovem, pelo menos no que se refere a frases simples.

According to this interpretation the presence of a P600 effect in 2-year- olds would mean that late integration processes are already at work at this age even though highly automatic syntactic processes reflected in the ELAN are not yet effective. The biphasic ELAN-P600 pattern observed in 2.8-year-olds indicates that local phrase 
structure building processes are already established by that age in addition to late integration processes at least for the processing of simple active sentences. A similar developmental shift from a P600-only to a biphasic ELAN-P600 pattern was reported for the processing of phrase structure violations in passive sentences between the age of 6 and 7 years (Hahne, Eckstein, and Friederici, 2004). (FRIEDERICI; THIERRY, 2008, p. 227)

Em 2010, Bernal e colegas estudaram se as crianças construíam expectativas online em relação à categoria sintática da próxima palavra em uma frase. Os pesquisadores apresentaram frases que tinham substantivos no lugar de verbos e vice-versa. Os resultados mostraram respostas cerebrais diferentes para sentenças gramaticais e não-gramaticais, sugerindo que aos 24 meses de idade, as crianças constroem expectativas on-line sobre a categoria das palavras. Outro achado interessante foi que a topografia da resposta cerebral foi diferente para substantivos e verbos, sugerindo que o processamento de substantivos e verbos em crianças pequenas já poderia ser conduzido por mecanismos subjacentes diferentes (como é encontrado em adultos).

Em 2012, o mesmo grupo relatou resultados semelhantes com novas palavras (BRUSINI, 2012 - tese de doutorado). Eles ensinaram às crianças substantivos e verbos novos ${ }^{5}$ criados para o teste com a fonotática do francês, touse como em (4), uma semana antes do teste - nunca com a estrutura alvo -, e, no dia do teste, como em Bernal et al. (2010), eles os apresentaram em frases gramaticais como em (5) ou agramaticais nas quais os substantivos ensinados na semana anterior eram inseridos agora em estrutura verbal, como em (6), e vice-versa.

(4) Oh regarde, un touse!

Ih Olha, um touse!

(5) L'indien pousse le [touse $]_{\mathrm{N}}$

O índio empurra o touse.

\footnotetext{
${ }^{5}$ As palavras inventadas eram ensinadas a partir de brincadeiras com bonecos. Touse, por exemplo, foi ensinado com o significado de tricerátopes.
} 
(6) *Alors, elle le [touse $]_{\mathrm{V}}$ de joie.

Então, ela o touse de alegria.

Os pesquisadores também encontraram respostas diferentes para frases gramaticais e agramaticais, embora com uma topografia e uma janela temporal diferentes do que aquelas do estudo de 2010. Essa tese se converteu em um número de artigos publicados em 2016 - BRUSINI et al., 2016a, 2016b).

Ainda em relação ao processamento sintático, e mais especificamente aos verbos e à morfossintaxe, em 2005, dois artigos do mesmo grupo de pesquisadores surgiram. O estudo com crianças mais velhas foi publicado em primeiro lugar e, em seguida, um grupo mais jovem também foi testado e os resultados desses testes foram publicados em um artigo separado. Isso significa que, uma vez que os autores observaram os potenciais obtidos pela manipulação de propriedades morfossintáticas e semânticas, eles estavam confiantes de que tal ativação poderia ser encontrada em crianças mais novas. É comum na literatura do ERP de desenvolvimento procurar primeiro os fenômenos em um grupo etário e, em seguida, tentar observar quão cedo pode-se observar o fenômeno. Silva-Pereyra et al. (2005b) abordaram o curso temporal dos ERPs utilizando frases sintáticas e semanticamente anômalas para comparar com as controle, com crianças de 36 meses de idade (SILVAPEREYRA; RIVERA-GAXIOLA; KUHL, 2005b). O objetivo era observar que tipo de ativação cerebral seria observada quando sentenças sintática e semanticamente anômalas foram apresentadas aos participantes em idade pré-escolar. Silva-Pereyra et al. (2005a) apresentaram o mesmo conjunto de estímulos em uma população mais jovem, de 30 meses de idade (SILVA-PEREYRA et al., 2005a).

Silva-Pereyra e colaboradores estudaram crianças de 36 e 48 meses, manipulando elementos sintáticos e semânticos (SILVAPEREYRA, RIVERA-GAXIOLA, KUHL, 2005). Eles adicionaram o sufixo -ing às estruturas em que ele não é licenciado como em (7) e alteraram o argumento interno dos verbos tornando-os agramaticais, como em (8).

(7) My uncle will watching a movie.

1POS tio FUT assistir-ASP DET filme

Meu tio irá assistindo um filme. 
(8) My uncle will blow the movie.

1POS tio FUT soprar DET filme

Meu tio vai soprar um filme.

Com essa manipulação, os autores demonstraram que crianças de 36 e 48 meses apresentaram efeitos ERP diferentes para processamento sintático e semântico (mesmo sem atenção explícita às frases) e que suas respostas mudaram entre 3 e 4 anos de idade. As ativações tiveram localização mais anterior aos 3 do que aos 4 anos de idade. $\mathrm{O}$ mesmo padrão foi replicado em crianças de 2,5 anos de idade.

Eles descobriram uma mudança positiva significativa na forma de onda durante o processamento de frases sintaticamente anômalas quando comparadas com frases não anômalas. Este efeito foi composto apenas de um efeito positivo duradouro que foi maior sobre as regiões anteriores do escalpo de 500 a $1500 \mathrm{~ms}$. A interpretação foi que o efeito foi consistente com os resultados de P600 de adultos durante o processamento sintático. Os P600 adultos sob o mesmo tipo de manipulação são apresentados em eletrodos de linha média parietal (HAGOORT; BROWN; OSTERHOUT, 1999). Entretanto, o efeito sintático em pré-escolares tinha uma distribuição anterior. Uma possível conclusão após a análise desses resultados é que, já em tenra idade, as crianças têm a capacidade de analisar a sintaxe. Eles implementam diferentes processos ao analisar sentenças gramaticais e não-gramaticais. Em geral, o potencial evocado foi uma onda de positividade tardia. Este resultado foi interpretado como semelhante ao P600 adulto, mas os pré-escolares tiveram uma resposta mais distribuída nos eletrodos anteriores. O mesmo padrão estava presente em crianças de 2,5 anos (SILVA-PEREYRA et al., 2005a). A conclusão geral foi de que as crianças pequenas possuem assinaturas neurais do processamento das sentenças no início de seu desenvolvimento linguístico.

Em outro estudo dos mesmos autores (SILVA-PEREYRA et al., 2005b) são comparadas as ativações de crianças de 36 e 48 meses em frases com violações morfossintáticas e semânticas. O estudo demonstrou que crianças de 36 meses e 48 meses demonstram efeitos de ERP diferentes para processamento sintático e semântico sem atenção explícita às sentenças e que suas respostas mudam entre 3 e 4 anos de idade para as violações sintáticas. Uma onda positiva foi observada 
nas frases sintaticamente anômalas nos participantes de 36 meses com pico de ativação em $800 \mathrm{~ms}$ e duas ondas positivas na população de 48 meses: uma com pico aos $400 \mathrm{~ms}$ e a outra em $800 \mathrm{~ms}$. Essas ativações foram reportadas como P400 e P800. Os autores também reportaram uma diferença quanto à topografia, que demonstrou ativações mais fortes em regiões frontocentrais. Em relação às frases com anomalias semânticas, três ativações negativas foram reportadas como N400, N600 e N800, nos dois grupos.

Outra área de investigação na literatura da linguística e de processamento de frases é a importância das pistas sintáticas e semânticas. Essa importante questão também foi investigada em crianças. Para poder observar se as sugestões semânticas são mais salientes do que a estrutura das frases, Silva-Pereyra e colegas apresentaram a crianças de 36 meses com frases em inglês e em jabberwocky, ${ }^{6}$ sem conteúdo semântico - ou seja, usando apenas a estrutura de frases com palavras sem qualquer significado (SILVA-PEREYRA et al., 2007). Eles testaram 16 crianças. Metade das sentenças eram agramaticais. As frases usadas tinham verbos transitivos como em (9). No exemplo, o argumento interno (the cat) é um DP. Os pesquisadores substituíram o tipo correto de argumento interno, um DP, por um sintagma preposicional como em (10). Esta é uma violação da estrutura do verbo "touch". Nas frases jabberwocky a posição das palavras funcionais é respeitada em frases gramaticais como em (11) e (12), mas as palavras lexicais são substituídas por palavras possíveis mas inexistentes na língua.

(9) My dolly touched the cat with her hand.

'Minha bonequinha tocou o gato com a mão dela.'

(10) My dolly touched with the cat her hand.

'Minha bonequinha tocou com o gato a mão dela.'

(11) My cholly daunched the glat with her shond.

'Minha tonequinha docou o clato com a prão dela.'

\footnotetext{
${ }^{6}$ Jabberwocky é o termo para denominar sentenças com estruturas aparentemente perfeitas na língua mas que não fazem sentido algum. O termo é original do livro Through the Looking-Glass, and What Alice Found There, de Lewis Carrol (1871).
} 
(12) My cholly daunched with the glat her shond

'Minha tonequinha docou com o clato a prão dela.'

Se as crianças utilizassem o conhecimento semântico para determinar se uma frase era incongruente ou não, mais do que o conhecimento sintático, então seria de se esperar que os efeitos de ativação fossem mais fortes para frases que eram semanticamente incongruentes do que sintaticamente incongruentes. Os autores encontram ativações negativas e positivas maiores para as frases anômalas do que para as não-anômalas. No entanto, os resultados encontrados pelos autores são difíceis de interpretar dado que nenhum dos efeitos durou mais de $200 \mathrm{~ms}$. Na verdade, as ativações encontradas tinham apenas $150 \mathrm{~ms}$, o que é bastante rápido para esse tipo de fenômeno. Sendo assim, o que o leitor levo do artigo é a variedade de latência e localização que os autores encontraram que por vezes estavam alinhadas com outros estudos, mas também foram observadas latências e localização ainda não reportada.

O último estudo que será relatado diz respeito a uma investigação quanto à estrutura argumental. COSTA (2015) investigou se crianças de dois anos sabem inconscientemente que certos verbos entram em estruturas argumentais específicas como em (13), demonstrando efeito de agramaticalidade caso um verbo conhecido entrasse em outra estrutura como em (14), presente em outras línguas do mundo, mas não observada nos dados da língua francesa. Pequenos vídeos em que uma narradora contava uma história enquanto manipulava bonecos era apresentado às crianças. Ao longo das histórias, frases gramaticais - do tipo (13) e (15) e agramaticais como em (14) eram ouvidas e as pesquisadoras analisaram a ativação cerebral relacionada a essas frases. Os resultados demonstraram uma ativação negativa entre 200-450ms após a criança ter escutado o verbo que estava sendo manipulado. Esses resultados indicaram que as crianças são sensíveis a manipulações na estrutura argumental.

(13) Thomas lui donne une fleur pour lui dire merci.

'Thomas lhe deu uma flor para lhe dizer obrigado.'

(14) * Elle lui donne qu'ils seront toujours amis.

'Ela lhe deu que eles seriam sempre amigos.' 
(15) Elle lui crie qu'il faut manger la bonne viande. 'Ela lhe gritou que ele devia comer a boa carne.'

Todos esses estudos indicam que crianças bem pequenas que produzem palavras ou pequenas sentenças há poucos meses no momento dos testes já têm intuição linguística sobre o que é possível ou não em sua língua nativa quanto ao processamento de frases. Violações sintáticas e semânticas eliciam ondas diferentes daquelas encontradas em frases bem formadas. Os resultados apresentados aqui sugerem que crianças de um ano e meio, dois e três computam estruturas sintáticas complexas online. Isso nos leva a pensar sobre quais mecanismos já estão ativos nessa idade e a que outros tipos de subcategorização de verbos as crianças são sensíveis e quando essa(s) capacidade(s) estaria(m) disponível(is).

\section{Conclusão}

A ativação observada quando crianças processam sentenças não ocorre necessariamente na mesma topografia e janela temporal encontradas nos componentes clássicos de ERP. Alguns autores têm reportado ativações que ocorrem em janelas de 500-1500ms e em posições mais anteriores como P600. Isso estaria alinhado com o que Gouvea (2010) observa em sua revisão sobre o P600. No entanto, não sabemos dizer ainda se todos os elementos reportados como P600 em adultos vão ser encontrados na população infantil e se eles representarão os mesmos processos subjacentes. É importante dar continuidade às pesquisas para podermos, com um corpo maior de dados de outras línguas, conhecer mais sobre o processamento sintático infantil.

Aárea de estudo sobre desenvolvimento linguístico em ERP ainda é recente e as melhores formas de testar e interpretar os dados estão sendo continuamente debatidas, a fim de encontrar o melhor sistema. O campo está em ampla expansão e cada ano mais pesquisas e novos grupos são formados em todo o mundo. A comparação com adultos é necessária, mas às vezes, quando a ativação encontrada não está alinhada com aquela em adultos não há uma conclusão óbvia. A diferença pode existir devido ao nível de maturidade linguística ou fatores relacionados à anatomia infantil. Sendo assim, dizer apenas que os resultados são diferentes não necessariamente nos leva a conclusões claras sobre quais processos tal ativação indica. 
Mesmo assim, o que sabemos hoje sobre o processamento infantil com ERP já é bastante encorajador. Crianças de 18 meses são sensíveis a manipulações quanto a categoria em palavras do dia-a-dia e aos 24 meses em palavras aprendidas no laboratório. Estudos mostram que, aos 24 meses, as crianças são sensíveis a manipulações quanto à marcação de caso e que aos 28 meses a ativação encontrada já apresenta padrão adulto. Aos 30 meses, as crianças são sensíveis a manipulações sintáticas e semânticas. Essa mesma manipulação elicia uma ativação com localização mais anterior em crianças de 3 do que em crianças de 4 anos. Pesquisadores também encontraram diferenças de ativação em crianças de 36 meses quando frases jabberwocky foram testadas vs. frases padrão das línguas.

Neste artigo, foi apresentada a técnica de ERP, mostrando o caminho que o campo tomou ao tentar entender os mecanismos subjacentes à cognição e especificamente à linguagem - a literatura adulta foi brevemente apresentada, mostrando seus principais componentes e estudos mais relevantes. Em seguida, apresentamos a literatura sobre o desenvolvimento infantil utilizando a técnica de ERP e seus desafios e resultados encorajadores. No final do artigo, os artigos mais importantes sobre a estrutura de argumentos usando a técnica ERP também foram resumidos. A ativação observada quando as crianças processam sentenças não ocorre necessariamente na mesma topografia e janela temporal encontradas em componentes clássicos de ERP. Alguns autores relataram ativações ocorrendo em janelas de $500-1500 \mathrm{~ms}$ e em posições anteriores como P600. Isso estaria em consonância com o que Gouvea et al. (2010) observa em sua revisão da P600. No entanto, ainda não sabemos se todos os elementos relatados, como P600 em adultos, serão encontrados na população infantil e se eles representarão os mesmos processos subjacentes. É importante continuar a pesquisa para que possamos ter um corpo maior de dados de outros laboratórios, outras tarefas e línguas para saber mais sobre o processamento sintático das crianças.

Sendo assim, este campo de investigação tem grandes desafios a enfrentar ao tentar abranger diferentes fontes de informação de diferentes disciplinas relacionadas ao estudar a linguagem e/ou o desenvolvimento infantil. Mesmo assim, estou otimista de que a fertilização mútua (crossfertilization) é possível (POEPPEL, EMBICK, 2005), se tentarmos pesquisar fenômenos com a devida diligência. No entanto, não devemos ser afoitos em supor que uma ativação cortical em crianças é diretamente comparável aos resultados encontrados em adultos. Até agora, ainda 
precisamos de mais pesquisas para ter certeza de que as mesmas janelas de tempo e polaridade significam as mesmas computações para ambas populações.

\section{Agradecimentos}

Parte desta pesquisa foi conduzida com o apoio do CNPq e Fundação CAPES (Bolsista CAPES no Processo 17638-12-7), que apoiou um ano de trabalho em Paris, no Laboratoire de Science Cognitive et Psycholinguistique (LSCP) na École Normale Superieure, Paris, França. Gostaria também de agradecer as minhas orientadoras Professoras Doutoras Aniela Improta França e Anne Christophe pelo suporte durante o período do meu doutorado e para além desse momento de formação acadêmica bem como toda a equipe dos laboratórios Acesin e LSCP.

\section{Referências}

BERKUM, J.J.A.; HAGOORT, P. ; BROWN, C. M. Semantic Integration in Sentences and Discourse: Evidence from the N400. Journal of Cognitive Neuroscience, MIT, v.11:6, p. 657-671, 1999. https://doi. org/10.1162/089892999563724.

BERNAL, S.; DEHAENE-LAMBERTZ, G.; MILlotTE, S.; CHRISTOPHE, A. Two-year-olds compute syntactic structure on-line. Developmental Science, Wiley Online Library, v. 13, n. 1, p. 69-76, jan. 2010. ISSN 1467-7687.

BORNKESSEL-SCHLESEWSKY, I.; SCHLESEWSKY,M. An Alternative perspective on "semantic P600" effects in language comprehension, Brain Research Reviews, Elsevier, v.59, p. 55-73, 2008. https://doi.org/10.1016/j.brainresrev.2008.05.003.

BROWN, C.; HAGOORT, P.; KUTAS, M. Postlexical Integration Processes in Language Comprehension: Evidence from BrainImaging Research. In: GAZZANIGA, M. S. (Ed.). The New Cognitive Neurosciences. [S.1.]: A Bradford Book; MIT Press, 2000. cap. 61, p. 881-895. ISBN 0-262-07195-9. 
BRUSINI, P. Découvrir les noms et les verbes: Quand les classes sémantiques initialisent les catégories syntaxiques. 2012. Tese (Doutorado) - École Normale Superieure, França, 2012.

BRUSINI, P.; DEHAENE-LAMBERTZ, G.; van HEUGTEN, M.; CARVAlHO, A.; GOFFINET, F.; FIÉVET, A. C.; CHRISTOPHE, A. Ambiguous function words do not prevent 18-month-olds from building accurate syntactic category expectations: an ERP study. Neuropsychologia, Elsevier. In Press. https://doi.org/10.1016/j. neuropsychologia.2016.08.015.

BRUSINI, P.; DEHAENE-LAMBERTZ, G.; DUTAT, M.; GOFFINET, F.; CHRISTOPHE, A. ERP evidence for on-line syntactic computations in 2-year-olds. Developmental Cognitive Neuroscience, Elsevier, v. 19, p. 164-173, 2016a. https://doi.org/10.1016/j.den.2016.02.009.

BRUSINI, P. DEHAENE-LAMBERTZ, G.; DUTAT, M.; GOFFINET, F.; CHRISTOPHE, A. ERP evidence for on-line syntactic computations in 2-year-olds, Developmental Cognitive Neuroscience, Elsevier, v.19, p. 164-173, jun. 2016b. https://doi.org/10.1016/j.dcn.2016.02.009.

COCH, D.; MITRA, P.; GEORGE, E. Behavioral and ERP evidence of word and pseudoword superiority effects in 7- and 11-year-olds. Brain Research, Elsevier, v. 1486, p. 68-81, nov. 2012. ISSN 1872-6240.

CONBOY, B. T.; RIVERA-GAXIOLA, M.; SILVA-PEREYRA, J. F.; AND KUHL, P. K. Event-related potential studies of early language processing at the phoneme, word, and sentence levels. In: FRIEDERICI, A. D.; THIERRY, G. (Org.). Early language development: bridging brain and behavior, Trends in language acquisition research series. Amsterdam/ The Netherlands: John Benjamins, 2008. v. 5, p. 24-64. https://doi. org/10.1075/tilar.5.04con.

COSTA, M. U. C. L. M. Argument Stucture in Language Acquisition: an ERP Study. 2015. 174f. Tese (Doutorado em Linguística) -Faculdade de Letras, Universidade Federal do Rio de Janeiro, Rio de Janeiro, 2015.

COULSON, S.; KING, J. W.; KUTAS, M. Expect the Unexpected: Eventrelated Brain Response to Morphosyntactic Violations. Language and Cognitive Processes, Taylor \& Francis Online, v. 13, n. 1, p. 21-58, 1998. ISSN 0169-0965. 
DEBOER, T.; SCOTT, L.; NELSON, C. Methods for acquiring and analyzing infant event-related potentials. In: HAAN, M. D. (Org.). Infant EEG and event-related potentials. [S.1.]: Psychology Press, 2007. cap. 1, p. 5-38.

DEBRUILLE, J. B. The N400 potential could index a semantic inhibition. Brain Research Reviews, Elsevier, v. 56, n. 2, p. 472-427, dez. 2007. ISSN 0165-0173.

DEHAENE-LAMBERTZ, G.; PENA, M. Electrophysiological evidence for automatic phonetic processing in neonates. Neuroreport, LWW Journals, v. 12, n. 14, p. 3155-3158, out. 2001. ISSN 0959-4965.

FRANCA, A. I. et al. Discriminating among different types of verbcomplement merge in Brazilian Portuguese: An ERP study of morphosyntactic sub-processes. Journal of Neurolinguistics, Elsevier, v. 17, p. 425-437, 2004. ISSN 09116044.

FRANÇA, A. I. Concatenações linguísticas: estudo de diferentes módulos cognitivos na aquisição e no córtex. 236f. 2002. Tese (Doutorado) Universidade Federal do Rio de Janeiro, Rio de Janeiro, 2002.

FRANÇA, A; GOMES, J. Técnica de ERP: investigando a assimetria sujeito-objeto na interface sintaxe-semântica com EEG. Letras de Hoje, Porto Alegre, v. 50, n. 3, p. 360-370, jul.-set. 2015. https://doi. org/10.15448/1984-7726.2015.3.18411.

FRANÇA, A.I.; LEMLE, M; GESUALDI, A.; CAGY, M.; INFANTOSI, A.F.C. A neurofisiologia do acesso lexical: palavras em português. Revista Veredas, Juiz de Fora, UFJF, v. 2, p. 34-49, 2008.

FRIEDERICI, A. D. The time course of syntactic activation during language processing: a model based on neuropsychological and neurophysiological data. Brain and Language, Elsevier, v. 50, p. 259-281, 1995. https://doi.org/10.1006/brln.1995.1048

FRIEDERICI, A. D. Towards a neural basis of auditory sentence processing. Trends in Cognitive Sciences, Elsevier, v. 6, n. 2, p. 78-84, fev. 2002. ISSN 1364-6613.

FRIEDERICI, A. D. et al. Processing linguistic complexity and grammaticality in the left frontal cortex. Cerebral Cortex [New York, N.Y., 1991], Oxford Academic, v. 16, n. 12, p. 1709-1717, dez. 2006. ISSN 1047-3211. 
FRIEDERICI, A. D.; FRIEDRICH, M.; CHRISTOPHE, A. Brain responses in 4-month-old infants are already language specific. Current Biology: CB, Bethesda, v. 17, n. 14, p. 1208-1211, jul. 2007. ISSN 09609822.

FRIEDERICI, A. D.; FRISCH, S. Verb Argument Structure Processing: The Role of Verb Specific and Argument-Specific Information. Journal of Memory and Language, Elsevier, v. 43, n. 3, p. 476-507, out. 2000. ISSN 0749596X.

FRIEDERICI, A. D. et al. Syntactic parsing preferences and their on-line revisions: A spatio-temporal analysis of event-related brain potentials. Cognitive Brain Research, Bethesda, v. 11, p. 305-323, 2001. ISSN 09266410.

FRIEDERICI, A. D.; MEYER, M. The brain knows the difference: two types of grammatical violations. Brain Research, v. 1000, n. 1-2, p. 72-77, mar. 2004. ISSN 0006-8993.

FRIEDERICI, A. D.; PFEIFER, E.; HAHNE, A. Event-related brain potentials during natural speech processing: effects of semantic, morphological and syntactic violations. Cognitive Brain Research, Bethesda, v. 1, n. 3, p. 183-192, out. 1993. ISSN 09266410.

FRIEDERICI, A. D.; THIERRY, G. (Org.). Early Language Development. Bridging brain and behaviour. (Trends in Language Acquisition Research) Amsterdam/Philadelphia, John Benjamins Publishing Company, 2008. https://doi.org/10.1075/tilar.5.

FRIEDERICI, A. D.; WEISSENBORN, J. Mapping sentence form onto meaning: the syntax-semantic interface. Brain research, Elsevier, v. 1146, p. 50-58, maio 2007. ISSN 0006-8993.

FRIEDRICH, M.; FRIEDERICI, A. N400-like semantic incongruity effect in 19-month-old infants: Processing known words in picture contexts. Journal of cognitive neuroscience, p. 1465-1477, 2004. https://doi.org/10.1162/0898929042304705.

FRIEDRICH, M.; FRIEDERICI, A. D. Maturing brain mechanisms and developing behavioral language skills. Brain and Language, Elsevier, v. 114, n. 2, p. 66-71, 2010. ISSN 0093934X. 
FRISCH, S.; HAHNE, A.; FRIEDERICI, A. D. Word category and verbargument structure information in the dynamics of parsing. Cognition, Elsevier, v. 91, n. 3, p. 191-219, abr.2004. ISSN 0010-0277.

GESUALDI, A. DA R.; FRANÇA, A. I. Event-related brain potentials (ERP): an overview. Revista LinguiStica, UFRJ, Rio de Janeiro, v. 7, n. 2, p. 24-42, 2011. 39.

GIBSON, E. Linguistic complexity: locality of syntactic dependencies. Cognition, Elsevier, v. 68, p. 1-76, 1998. ISSN 00100277.

GOMES, J. N. Investigating the dissociation between $n 400$ and $p 600$ effects on the syntax semantics interface: an erp study. $183 \mathrm{f}$. Tese (Doutorado) - Faculdade de Letras da UFRJ, Rio de Janeiro, 2014.

GOMES, J. N.; FRANÇA, A. I. The ample semantic scope of minute language computations: an ERP study of words in Portuguese. Revista da ANPOLL, Anpoll, v. 34, p. 309-346, 2013. Disponível em: $<$ http://anpoll. emnuvens.com.br/revista/article/view/673/698 45>.

GOMES, J.; FRANÇA, A. I. A técnica de ERP: Investigando a assimetria sujeito-objeto na interface sintaxe-semântica com EEG. Letras de Hoje (Online), PUCRS, Porto Alegre, v. 4, p. 152-163, 2015.

GOUVEA, A. C. et al. The linguistic processes underlying the P600. Language and Cognitive Processes, Taylor \& Francis Online, v. 25, n. 2, p. 149-188, fev. 2010. ISSN 0169-0965.

HAGOORT, P. How the brain solves the binding problem for language: a neurocomputational model of syntactic processing. Neuroimage, Elsevier, v. 20, p. 18-29, 2003. https://doi.org/10.1016/j.neuroimage.2003.09.013.

HAGOORT, P. On Broca, brain, and binding: a new framework. Trends in cognitive sciences, Elsevier, v. 9, n. 9, p. 416-423, set. 2005. ISSN 1364-6613.

HAGOORT, P.; BROWN, C.; GROOTHUSEN, J. The syntactic positive shift (SPS) as an ERP measure of syntactic processing. Language and Cognitive Processes, Taylor \& Francis Online, v. 8, n. 912873514 , p. 439-483, 1993. ISSN 0169-0965.

HAGOORT, P.; BROWN, C.; OSTERHOUT, L. The neurocognition of syntactic processing. In: BROWN, C. M.; HAGOORT, P. (Org.). The neurocognition of language. Oxford: Oxford University Press, 1999. p. 273-317. 
HAGOORT, P.; BROWN, C. M. ERP effects of listening to speech compared to reading: the P600 / SPS to syntactic violations in spoken sentences and rapid serial visual presentation. Neuropsychologia, Elsevier, v. 38, p. 1531-1549, 2000. https://doi.org/10.1016/S00283932(00)00053-1.

HAGOORT, P.; LEVELT, W. The speaking brain. Science, American Association for the Advancement of Science, v. 326, p. 372-373, 2009. https://doi.org/10.1126/science.1181675.

HÄNEL-FAULHABER, B.; SKOTARA, N.; KÜGOW, M.; SALDEN, U.; BOTTARI, D.; RÖDER, B. ERP correlates of German Sign Language processing in deaf native signers. BMC Neuroscience, Springer Nature, v.15, n. 62, p. 1-11, 2014.

HERTEN, M. van; KOLK, H. H. J.; CHWILLA, D. J. An ERP study of P600 effects elicited by semantic anomalies. Brain research. Cognitive Brain Research, Bethesda, v. 22, n. 2, p. 241-255, mar. 2005. ISSN 09266410.

HOEKS, J. C. J.; STOWE, L. a.; DOEDENS, G. Seeing words in context: the interaction of lexical and sentence level information during reading. Brain Research. Cognitive Brain Research, Bethesda, v. 19, n. 1, p. 59-73, mar. 2004. ISSN 0926-6410.

HOLCOMB, P.; COFFEY, S.; NEVILLE, H. Visual and auditory sentence processing: A developmental analysis using event-related brain potentials. Developmental Neuropsychology, Taylor \& Francis Online, v. 8, p. 203-312, 1992. https://doi.org/10.1080/87565649209540525.

HWANG, H.; STEINHAUER, K. Phrase length matters: the interplay between implicit prosody and syntax in Korean "garden path" sentences. Journal of Cognitive Neuroscience, MIT Press, v. 23, n. 11, p. 3555-3575, nov. 2011. ISSN 1530-8898.

JOHNSON, M. Functional Brain Development During Infancy. In: BREMMER, G.; FOGEL, A. (Ed.). Blackwell handbook of infant development. [S.1.]: Blackwell Publishing, 2001. cap. 6.

KAAN, E. et al. The P600 as an index of syntactic integration difficulty. Language and Cognitive Processes, Taylor \& Francis Online, v. 15, n. 2, p. 159-201, abr. 2000. ISSN 0169-0965. 
KIM, A.; OSTERHOUT, L. The independence of combinatory semantic processing: Evidence from event-related potentials. Journal of Memory and Language, Elsevier, v. 52, n. 2, p. 205-225, fev. 2005. ISSN 0749596X.

KOLK, H. H. J. et al. Structure and limited capacity in verbal working memory: a study with event-related potentials. Brain and language, Elsevier, v. 85, p. 1-36, 2003.

KOOIJMAN, V. et al. Predictive brain signals of linguistic development. Frontiers in Psychology, Frontiers Media, v. 4, p. 25, Feb. 2013. ISSN 1664-1078. https://doi.org/10.3389/fpsyg.2013.00025.

KOTZ, S. A.; HOLCOMB, P. J.; OSTERHOUT, L. ERPs reveal comparable syntactic sentence processing in native and non-native readers of English. Acta Psychologica, Elsevier, v. 128, n. 3, p. 514-527, jul. 2008. ISSN 0001-6918.

KOVELMAN, I. Neuroimaging Methods. In: HOFF, E. Research Methods in Child Language: A Practical Guide. [S.1.]: Wiley-Blackwell, 2012. Cap. 4, p. 44-59.

KUPERBERG, G.; SITNIKOVA, T. Electrophysiological distinctions in processing conceptual relationships within simple sentences. Cognitive Brain Research, Bethesda, v. 17, p. 117-129, 2003. https://doi.org/10.1016/S0926-6410(03)00086-7.

KUPERBERG, G. R. Neural mechanisms of language comprehension: challenges to syntax. Brain Research, Elsevier, v. 1146, p. 23-49, maio 2007. ISSN 0006-8993.

KUPERBERG, G. R. et al. Building up linguistic context in schizophrenia: evidence from self-paced reading. Neuropsychology, Elsevier, v. 20, n. 4, p. 442-452, jul. 2006. ISSN 0894-4105.

KUTAS, M.; DELONG, K. A Sampler of Event-related brain Potential (ERP) Analyses of Language Processing. Brain Research in Language, Springer, v. 1, p. 153-186, 2008. https://doi.org/10.1007/978-0-38774980-8_6.

KUTAS, M.; FEDERMEIER, K. D. Thirty years and counting: finding meaning in the N400 component of the event-related brain potential (ERP). Annual Review of Psychology, Annual Reviews, v. 62, p. 621-647, jan. 2011. ISSN 1545-2085. 
KUTAS, M.; HILLYARD, S. Reading senseless sentences: Brain Potentials Reflect Semantic Incongruity. Science, American Association for the Advancement of Science, v. 207, n. 4427, p. 203-205, jan. 1980. https://doi.org/10.1126/science.7350657.

LAGE, A. C. Aspectos neurofisiológicos de concatenação e idiomaticidade em português do Brasil: um estudo de potenciais bioelétricos relacionados a eventos lingüísticos (ERPs). 2005. Tese (Doutorado em Lingüística) Universidade Federal do Rio de Janeiro, Rio de Janeiro, 2005.

LAU, E. F.; PHILLIPS, C.; POEPPEL, D. A cortical network for semantics: (de)constructing the N400. Nature Reviews. Neuroscience, Macmillan Publishers, v. 9, n. 12, p. 920-933, dez. 2008. ISSN 14710048 .

LUCK, S. J. An Introduction to the Event-Related Potential Technique. [S.1.]: MIT Press, 2005. ISSN 00393665.

MARANTZ, A. Generative linguistics within the cognitive neuroscience of language. The Linguistic Review, De Gruyter, n. 22, p. 429-445, Jan. 2005. ISSN 0167-6318.

MILLS, D. L. et al. Language experience and the organization of brain activity to phonetically similar words: ERP evidence from 14- and 20-month-olds. Journal of Cognitive Neuroscience, MIT Press, v. 16, n. 8, p. 1452-1464, out. 2004. ISSN 0898-929X.

NELSON, C.; LUCIANA, M. Handbook of Developmental Cognitive Neuroscience. 2nd. ed. [S.1.]: MIT Press, 2008. ISBN 9780262141048.

NEVINS, A. et al. The role of feature-number and feature-type in processing Hindi verb agreement violations. Brain Research, Elsevier, v. 1164, p. 81-94, 2007. https://doi.org/10.1016/j.brainres.2007.05.058.

OBERECKER, R.; FRIEDERICI, A. D. Syntactic event-related potential components in 24-month-olds' sentence comprehension. Neuroreport, LWW Journals, v. 17, n. 10, p. 1017-1021, jul. 2006. ISSN 0959-4965.

OBERECKER, R.; FRIEDRICH, M.; FRIEDERICI, A. D. Neural correlates of syntactic processing in two-year-olds. Journal of Cognitive Neuroscience, MIT Press, v. 17, n. 10, p. 1667-1678, out. 2005. ISSN 0898-929X. 
OSTERHOUT, L.; HOLCOMB, P. J. Event-related brain potentials elicited by syntactic anomaly. Journal of Memory and Language, Elsevier, v. 31, n. 6, p. 785-806, dez. 1992. ISSN 0749596X.

OSTERHOUT, L.; HOLCOMB, P. J.; SWINNEY, D. a. Brain potentials elicited by garden-path sentences: evidence of the application of verb information during parsing. Journal of Experimental Psychology. Learning, Memory, and Cognition, Washington, v. 20, n. 4, p. 786-803, jul. 1994. ISSN 0278-7393.

OSTERHOUT, L.; KIM, A.; KUPERBERG, G. The neurobiology of sentence comprehension. In: SPIVEY, M.; JOANNISSE, M.; MCRAE, K. (Org.). The Cambridge Handbook of Psycholinguistics. Cambridge: Cambridge University Press, 2007. p. 1-23.

OSTERHOUT, L.; MCLAUGHLIN, J. Novice Learners, Longitudinal Designs, and Event-Related Potentials: A Means for Exploring the Neurocognition of Second Language Processing. Language Learning, Wiley Online Library, v. 56, Supplement 1, p. 4-25, 2006.

PHILLIPS, C.; KAZANINA, N.; ABADA, S. H. ERP effects of the processing of syntactic long distance dependencies. Brain research. Cognitive Brain Research, Bethesda, v. 22, n. 3, p. 407-28, mar. 2005. ISSN 0926-6410.

PICTON, T. W. et al. Guidelines for using human event-related potentials to study cognition: recording standards and publication criteria. Psychophysiology, Wiley Online Library, v. 37, n. 2, p. 127-152, mar. 2000. ISSN 0048-5772.

POEPPEL, D.; EMBICK, D. Defining the relation between linguistics and neuroscience. In: CUTLER, A. (Ed.). Twenty-first century psycholinguistics: four cornerstones. Mahwah, N.J.: Lawrence Erlbaum, 2005.

POEPPEL, D.; OMAKI, A. Language acquisition and ERP approaches: Prospects and challenges. In: FRIEDERICI, A.; THIERRY, G. Early language development: bridging brain and behavior: Amsterdam: John Benjamins, 2008. https://doi.org/10.1075/tilar.5.12poe.

PUJOL, J. et al. Myelination of language-related areas in the developing brain. Neurology, American Academy of Neurology, v. 66, n. 3, p. 339343, fev. 2006. ISSN 1526-632X. 
PYLKKÄNEN, L.; BRENNAN, J.; BEMIS, D. Grounding the cognitive neuroscience of semantics in linguistic theory. Language and Cognitive Processes, Taylor \& Francis Online, v. 26, n. 9, p. 1317-1337, 2011.

SHAFER, V.; GARRIDO-NAG, K. The neurodevelopmental bases of language. In: HOFF, E.; SHATZ, M. (Org.). Blackwell handbook of language Development. Oxford: Blackwel, 2007. cap. 2, p. 21-45. https://doi.org/10.1002/9780470757833.ch2.

SILVA-PEREYRA, J. et al. Grammatical processing without semantics? An event related brain potential study of preschoolers using jabberwocky sentences. Journal of Cognitive Neuroscience, MIT Press, v. 19, n. 6, p. 1050-1065, jun. 2007. ISSN 0898-929X.

SILVA-PEREYRA, J. F. et al. Sentence processing in 30-month-old children: an event-related potential study. Neuroreport, LWW Journals, v. 16, n. 6, p. 645-648, abr. 2005a. ISSN 0959-4965.

SILVA-PEREYRA, J. F.; RIVERA-GAXIOLA, M.; KUHL, P. K. An event-related brain potential study of sentence comprehension in preschoolers: semantic and morphosyntactic processing. Brain Research. Cognitive Brain Research, Elsevier, v. 23, n. 2-3, p.247-258, maio 2005 b. ISSN 0926-6410.

TEPLAN, M. Fundamentals of EEG measurement. Measurement Science Review, De Gruyter, v. 2, p. 1-11, 2002.

ZHANG, Y. et al. Syntax does not necessarily precede semantics in sentence processing: ERP evidence from Chinese. Brain and Language, Elsevier, v. 126, n. 1, p. 8-19, jul. 2013. ISSN 1090-2155.

YUAN, S.; FISHER, C. Really? She Blicked the Baby? Two-Year-Olds Learn Combinatorial Facts About Verbs. Psychological Science, Sage Journals, v. 20, n. 5, p. 619-626, 2009. 